\title{
On the phase interpolation problem - $A$ brief review and some new results
}

\author{
S C DUTTA ROY and SHAILEY MINOCHA \\ Department of Electrical Engineering, Indian Institute of Technology, \\ Hauz Khas, New Delhi 110016, India
}

\begin{abstract}
Reconstruction of a signal from the phase of its Fourier transform is important and useful in a variety of practical applications. In this paper, we give a brief review of the previous results and develop some new ones pertaining to the phase-only reconstruction problem. The review includes conditions under which the signal is uniquely specified by its Fourier transform phase and some algorithms for performing the reconstruction. New results consist of an extension of a previous technique and an explicit formula for reconstruction. A number of potential applications of the reconstruction techniques are briefly mentioned in the concluding section.
\end{abstract}

Keywords. Signal reconstruction; phase interpolation; Fourier transform.

\section{Introduction}

In a variety of practical applications, it is required to reconstruct a signal from its partial Fourier domain information (Oppenheim et al 1983). Such partial information may consist of the Fourier transform (FT) phase alone, the FT magnitude alone (Fienup 1978; Hayes et al 1980a; Hayes 1982), or the signed FT magnitude (Van Hove et al 1983 ) (i.e. the FT magnitude together with a bipolar function, \pm 1 , representing one bit of phase information at each frequency). Signal reconstruction from FT phase alone is referred to as the magnitude-retrieval problem, and it is this problem that we address ourselves to in this paper.

It has been well recognized in the literature (Hayes et al 1980b; Oppenheim \& Lim 1981) that the phase of the FT of a signal contains a significant amount of information about the signal and that several important features of the signal are preserved if only the phase is retained. It is also well known that under certain conditions, phase information alone is sufficient to recover the original signal.

In general, of course, a signal is not uniquely defined by its phase ${ }^{1}$. This is well demonstrated by the fact that the phase remains invariant when a given signal is convolved with any zero-phase signal. Thus, whatever be the nature of the signal, it

\footnotetext{
${ }^{1}$ Throughout this paper, reference to the phase or magnitude of the signal should be interpreted, respectively, as the phase or magnitude of the FT of the signal.
} 
can, at best, be uniquely reconstructed from the phase information only to within an arbitrary zero-phase factor. Some constraints are therefore necessary to uniquely determine a signal from its phase. One such contraint is the minimum (or maximum) phase condition (Oppenheim \& Schafer 1975). For continuous-time signals with rational Laplace transforms, this condition corresponds to confining all the poles and zeros only to the left half (or right half) of the s-plane. For discrete-time signals with rational $z$-transforms, the corresponding confinement is within (or outside) the $z$-plane unit circle. Under these conditions, the Hilbert transform relation between the log-magnitude of the FT and its phase forms the basis of the conventional reconstruction algorithm. Alternatively, Quatieri \& Oppenheim (1981) have developed iterative algorithms for reconstructing a minimum (or maximum) phase signal from the phase or magnitude of its FT. These algorithms repeatedly impose the causality constraint in the time domain and incorporate the known phase or magnitude function in the frequency domain, the approach being similar to the iterative techniques proposed by Gerchberg \& Saxton (1972) and Fienup (1978). The convergence properties of these algorithms have been investigated in detail by Tom et al (1981). In addition, Yegnanarayana \& Dhayalan (1983) have proposed noniterative procedures for phase or magnitude-only reconstruction for a minimum phase signal, based on the relation between log magnitude and phase of such a signal through cepstral coefficients (Yegnanarayana 1981, 1982). These techniques, though free from the convergence problems of iterative algorithms, require the phase in the unwrapped form for the phase-only reconstruction. Phase unwrapping is a well-known difficult problem because of the need for detection of the discontinuities in the principal (wrapped) value of the phase (Oppenheim \& Schafer 1975; Tribolet 1977).

Many practical signals do not, however, satisfy the minimum or maximum phase condition. For some classes of them, conditions have been derived for recovery of the signal again, to within a scale factor from its phase. We summarize these conditions (Hayes et al 1980b) in the next section; for proofs and detailed discussions, reference may be made to Hayes et al (1980a), Oppenheim (1981), and Oppenheim et al (1982).

\section{Conditions for exact reconstruction from phase}

A basic result relating to exact reconstruction from phase can be stated in the form of the following theorem.

Theorem 1. A one-dimensional discrete-time signal which is of finite length and which has a z-transform with no zeros in conjugate reciprocal pairs or on the unit circle is uniquely specified to within a scale factor by the phase of its FT(or by the tangent of its phase).

While zeros on the unit circle are disallowed for convenience, zeros in conjugate reciprocal pairs are disallowed for avoiding possible ambiguity due to zero-phase components.

In theorem 1, the phase is assumed to be known for all frequencies. In a practical situation, however, only a finite set of samples of the phase will be known. Theorem 1 has therefore been extended to take care of this situation as follows.

Theorem 2. A one-dimensional discrete-time signal which is known to be zero outside the interval $0 \leqslant n \leqslant N$ is uniquely specified to within a scale factor by $N$ samples of its 
phase (or tangent of its phase) at distinct frequencies in the interval $0<\omega<\pi$ if it has a z-transform with no zeros on the unit circle or in conjugate reciprocal pairs.

This theorem forms the basis of several signal reconstruction algorithms discussed in the next section.

\section{Algorithms}

The problem of signal reconstruction from phase can be stated as follows: Let $\boldsymbol{P}_{N}(z)$ be the $z$-transform of a discrete-time finite duration signal $p^{(N)}(n), 0 \leqslant n \leqslant N$, i.e.

$$
P_{N}(z)=\sum_{n=0}^{N} p^{(N)}(n) z^{-n}
$$

The FT of the signal is simply obtained by replacing $z$ by $e^{j \omega}$ in (1). Let

$$
P_{N}\left(e^{j \omega}\right)=\left|P_{N}\left(e^{j \omega}\right)\right| e^{j \phi(\omega)}
$$

and let $\phi\left(\omega_{k}\right)=\phi_{k}$ be specified at $N$ distinct frequencies: $\omega_{k}, k=1,2, \ldots, N$, where $0<\omega_{k}<\pi$ and $\omega_{k}$ 's need not be ordered or uniformly spaced. It is assumed that $p^{(N)}(0) \neq 0$ and $P_{N}(z)$ does not have zeros on the unit circle or in conjugate reciprocal pairs implying that the phase values, $\phi_{k}$, do not contain a linear phase component. The assumption $p^{(N)}(0) \neq 0$ is made to simplify the algorithms. The problem is to reconstruct the signal from the given frequency-phase pairs $\left(\omega_{k}, \phi_{k}\right)$.

\subsection{Equations to be solved}

From (2), we have

$$
\frac{P_{N}\left(e^{j \omega}\right)}{P_{N}\left(e^{-j \omega}\right)}=\frac{e^{j \phi(\omega)}}{e^{-j \phi(\omega)}}=\frac{\sum_{n=0}^{N} p^{(N)}(n) e^{-j n \omega}}{\sum_{n=0}^{N} p^{(N)}(n) e^{j n \omega}}
$$

On cross multiplication and simplification, we have

$$
\left.\sum_{n=0}^{N} p^{(N)}(n) e^{-1[m+\phi \phi(t)]}\right]=\sum_{n=0}^{N} p^{(N)}(n) e^{j[n(s)+\phi(\omega)]}
$$

Equation (4) is satisfied only if the imaginary part of either side vanishes; this gives

$$
\sum_{n=0}^{N} p^{(N)}(n) \sin [n \omega+\phi(\omega)]=0 .
$$

Sampling $\phi(\omega)$ at $N$ distinct frequencies, $\omega_{k}, k=1,2, \ldots, N$ reduces (5) to the following set of $N$ linear equations

$$
\sum_{n=1}^{N} p^{(N)}(n) \sin \left(n \omega_{k}+\phi_{k}\right)=-p^{(N)}(0) \sin \phi_{k}, \quad k=1,2, \ldots, N
$$

$p^{(N)}(0)$ is, however, not known; the equations can be solved for $p^{(N)}(n) / p^{(N)}(0)$ which is equivalent to choosing $p^{(N)}(0)=1$ in $(6)$. The solution can be effected through any one of the well known algorithms for matrix inversion. 
Note that however exact the method may be, large $N$ will always lead to numerical instability and severe round-off errors. As a result, the applicability of this technique will be limited in practice.

To circumvent the problem, two iterative algorithms are available in the literature, which we now describe.

\subsection{First iterative algorithm}

This algorithm (Hayes et al 1980a; Oppenheim \& Lim 1981; Oppenheim et al 1982) involves going back and forth between the time and frequency domains with the relevant constraints imposed in each domain. Let the $M$-point DFT of $p^{(N)}(n)$, with $M \geqslant 2 N$ be denoted by

$$
P(k)=\left.P_{N}(\omega)\right|_{\omega=2 \pi k / M}=|P(k)| e^{j \phi(k)} .
$$

The iterative procedure begins with an initial guess $\left\{P_{0}(k) \mid\right.$ of the unknown DFT magnitude $|P(k)|$. Combining this with the given phase samples $\phi(k)$, and taking the $M$-point IDFT gives the first estimate $p_{1}(n)$,

$$
p_{1}(n)=\operatorname{IDFT}\left[\left|P_{0}(k)\right| e^{j \phi(k)}\right],
$$

$p_{1}(n)$ will be an $M$-point sequence. Since our aim is to obtain an $N+1$ point sequence, $p_{1}(n)$ is modified to another sequence $y_{1}(n)$ as follows.

$$
y_{1}(n)=\left\{\begin{array}{l}
p_{1}(n) \text { for } 0<n \leqslant N, \\
\beta \text { (arbitrary) for } n=0, \\
0 \text { for } N+1 \leqslant n \leqslant M-1
\end{array}\right.
$$

The DFT of $y_{1}(n), Y_{1}(k)$, is obtained and the second estimate of $p(n)$ is obtained as

$$
p_{2}(n)=\operatorname{IDFT}\left[\left|Y_{1}(k)\right| e^{j \phi(k)}\right] \text {. }
$$

Equations (7), (8), and (9) complete one iteration, and this process is repeated. It has been observed empirically, and also proved theoretically (Tom et al 1981), that the algorithm always converges, although the number of iterations may be large for large $N$. Since the DFT and IDFT are the major computational units, this algorithm does not suffer from numerical instability or round-off errors that arise in solving the set of equations (6) through matrix inversion.

The computation time required in this algorithm can be saved considerably by improving the convergence characteristics. This is precisely effected in the second iterative algorithm (Tom et al 1981; Oppenheim et al 1982) described below.

\subsection{Second iterative algorithm}

A compact representation of the first iterative algorithm is as follows.

where

$$
\mathbf{p}_{q+1}=T \mathbf{p}_{q}
$$

and

$$
\mathbf{p}_{q}=\left[p_{q}(0), p_{q}(1), \ldots, p_{q}(M)\right]^{t}
$$

$$
\mathbf{p}_{q+1}=\left[p_{q+1}(0), p_{q+1}(1), \ldots, p_{q+1}(M)\right]^{\ell}
$$


represent the estimates of the unknown vector $\mathbf{p}=[p(0), p(1), \ldots, p(M)]^{t}$ after the $q$ th and $(q+1)$ th iterations, respectively, and $T$ is a nonlinear operator corresponding to time limitation and phase substitution operations. It has been shown that convergence is improved if instead of (11), the iteration is modified to the following

$$
\mathbf{p}_{q+1}=\left(1-x_{q}\right) \mathbf{p}_{q}+x_{q} T \mathbf{p}_{q},
$$

where $\alpha_{q}$ is a scalar, referred to as the relaxation parameter, which may change with $q$. This is the second iterative algorithm. Note that for any choice of $\alpha_{q}$, the phase associated with $\mathbf{p}_{q+1}$ is the same as that of $\mathbf{p}$, by virtue of the definition of the operator $T$. Hence under appropriate conditions, a convergent solution of (12) will correspond to a scaled version of $\mathbf{p}$.

The question of convergence of (12) has been analysed in detail by Tom et al (1981), who have also derived the required optimum $\alpha_{q}$ for maximum rate of convergence of the iteration.

The problem of signal reconstruction has also been dealt with in terms of specified group delay functions (Yegnanarayana et al 1984). Based on the relation between the cepstral coefficients and the group delay functions of a signal, the authors have rederived the conditions for phase- (or magnitude-) only reconstruction. New iterative as well as noniterative techniques have been proposed and their convergence properties in the group delay domain have been extensively discussed (Yegnanarayana \& Dhayalan 1983; Yegnanarayana et al 1984; Madhu Murthy \& Yegnanarayana 1989). Applications of the proposed algorithms to speech and picture signals have also been demonstrated (Yegnanarayana et al 1984, 1988). In these studies, it has been recognized that the nature of the signal determines the relative importance of spectral magnitude or phase in its reconstruction. This contradicts the earlier notion (Oppenheim \& Lim 1981) that phase is more important than magnitude in all cases. The role of signal reconstruction from partial Fourier domain information and group delay functions in spectrum estimation and for sensor imaging applications have been discussed and illustrated in some recent contributions (Yegnanarayana et al 1990; Yegnanarayana \& Murthy 1990).

\subsection{Recursive algorithm}

In a recent contribution, Merchant (1988) has proposed a recursive technique for the reconstruction, which involves finding a polynomial $P_{N}(z)$, of order $N$, which satisfies phases at $N$ given frequencies.

He viewed the problem of reconstruction as that of determining a sequence of monic ${ }^{2}$ real polynomials $P_{m}(z)$, of order $m, m=2,3, \ldots, N$ satisfying the given frequencyphase characteristics on the unit circle, i.e.

$$
\operatorname{Phase}\left[P_{m}\left(e^{j \omega_{q}}\right)\right]=\phi_{q}, \quad q=1,2, \ldots, m .
$$

He showed that $P_{m}(z)$ is related to its predecessors $P_{m-1}(z)$ and $P_{m-2}(z)$ as follows

$$
P_{m}(z)=\alpha_{m-1} P_{m-1}(z)+\beta_{m-1}\left(z-2 \cos \omega_{m-1}+z^{-1}\right)\left[P_{m-1}(z)-P_{m-2}(z)\right],
$$

where $\alpha_{m-1}$ and $\beta_{m-1}$ are real numbers, and can be easily determined.

${ }^{2} \mathrm{~A}$ monic polynomial is defined as one in which the coefficient of the highest power term is unity. 
The coefficients of $P_{0}(z)$ and $P_{1}(z)$, required for initializing the recursion, are:

$$
\begin{aligned}
& p^{(0)}(0)=1, \\
& p^{(1)}(0)=1, \\
& p^{(1)}(1)=-\sin \phi_{1} / \sin \left(\omega_{1}+\phi_{1}\right) .
\end{aligned}
$$

Note that if $\omega_{1}+\phi_{1}=n \pi, n=0$ or any integer, then $p^{(1)}(1)$ cannot be computed. In such a case $\left(\omega_{1}, \phi_{1}\right)$ should be interchanged with some other $\left(\omega_{q}, \phi_{q}\right)$, such that $\omega_{q}+\phi_{q} \neq n \pi$.

Apart from the algorithms mentioned above, there is another solution to the problem of signal reconstruction from phase which is worth mentioning. Merchant (1982) reformulated the phase-reconstruction problem from time-domain considerations (see also Merchant \& Parks 1983). This gives rise to a system of equations whose coefficient matrix can be expressed as a sum (or difference) of a Toeplitz and a Hankel matrix. It has been shown by Merchant \& Parks (1982) that any matrix which can be expressed as a sum (or difference) of a Toeplitz and Hankel matrix can be converted to a block-Toeplitz form. Akaike (1973) has shown that a block-Toeplitz matrix can be inverted by a block-Levinson type recursion algorithm. Thus the system of equations can be solved in a very efficient manner. The applications of this technique and other algorithms discussed here will be given in $\$ 5$.

\section{Some new results}

We present in this section some new generalizations and extensions of the results of the previous section.

\subsection{Extension of Merchant's technique}

Merchant's (1988) recursive formulation, though very elegant, is not programmable on a computer. We have extended his technique for computer implementation (Minocha et al 1991). Here, we discuss the alogrithm and present several illustrative examples to demonstrate the accuracy and efficiency of our program ${ }^{3}$.

Substituting in (14) the corresponding polynomial expressions for $P_{m}(z), P_{m-1}(z)$ and $P_{m-2}(z)$ and carrying out somewhat lengthy algebraic manipulations results in the following recursive formulas for the coefficients of the polynomial:

where

$$
\begin{aligned}
p^{(m)}(r)= & \alpha_{m-1} p^{(m-1)}(r)+\beta_{m-1}\left[-2 \cos \omega_{m-1}\left(p^{(m-1)}(r)-p^{(m-2)}(r)\right)+\right. \\
& \left.\quad+\left(p^{(m-1)}(r-1)-p^{(m-2)}(r-1)\right)+\left(p^{(m-1)}(r+1)-p^{(m-2)}(r+1)\right)\right] \\
r= & 1,2, \ldots, m-1, \\
= & \beta_{m-1} p^{(m-1)}(m-1), r=m,
\end{aligned}
$$

$$
p^{(i)}(j)=0, \text { for } j>i \text {. }
$$

\footnotetext{
${ }^{3}$ The program, written in Fortran, may be obtained from the authors on request.
} 
The parameters $\beta_{m-1}$ and $\alpha_{m-1}$ in (18) are given by

where

$$
\begin{aligned}
& \beta_{m-1}=A_{m-1} / C_{m}, \\
& \alpha_{m-1}=1-\beta_{m-1}\left(p^{(m-1)}(1)-p^{(m-2)}(1)\right),
\end{aligned}
$$

$$
\begin{aligned}
& A_{m-1}=-\sin \phi_{m}-\sum_{r=1}^{m-1} p^{(m-1)}(r) \sin \left(r \omega_{m}+\phi_{m}\right) \\
& B_{m-1}=\sum_{r=1}^{m-2} p^{(m-2)}(r) \sin \left(r \omega_{m}+\phi_{m}\right)-\sum_{r=1}^{m-1} p^{(m-1)}(r) \sin \left(r \omega_{m}+\phi_{m}\right)
\end{aligned}
$$

and

$$
C_{m}=\left(p^{(m-1)}(1)-p^{(m-2)}(1)\right) A_{m-1}-2 B_{m-1}\left(\cos \omega_{m}-\cos \omega_{m-1}\right) .
$$

Merchant (1988) has cited two situations either of which will cause the recursion to terminate, and has also suggested remedial actions. We summarize his findings for the sake of completeness.

When $C_{m}=0, \alpha_{m-1}$ and $\beta_{m-1}$ cannot be determined [see (19)] and this brings the recursion to a halt. Thus, one needs to explore the conditions for which such a situation arises.

$C_{m}$ equals zero for the following cases:

Case 1: Consider a polynomial $H_{m}(z)$

$$
\begin{aligned}
H_{m}(z)= & P_{m-1}(z)\left[p^{(m-1)}(1)-p^{(m-2)}(1)\right]-\left(z-2 \cos \omega_{m-1}+z^{-1}\right) \times \\
& \times\left[P_{m-1}(z)-P_{m-2}(z)\right] .
\end{aligned}
$$

Note that the constant term of $H_{m}(z)$ is zero.

Also, $H_{m}(z)$ has the same structure as $P_{m}(z)$ in (14). Hence,

$$
\begin{aligned}
& \text { Phase }\left[H_{m}\left(e^{j \omega_{q}}\right)\right]=\phi_{q}, \quad q=1,2, \ldots, m-1, \\
& C_{m}=\operatorname{IM}\left[H_{m}\left(e^{j \omega_{m}}\right) e^{-\gamma \phi_{m}}\right],
\end{aligned}
$$

where $\mathrm{IM}[]$ is the imaginary part of [] .

$$
C_{m}=0 \text {, if and only if, }
$$

Phase $\left[H_{m}\left(e^{j \omega_{m}}\right)\right]=\phi_{m}$.

Equations (21) and (23) imply that

$$
\text { Phase }\left[H_{m}\left(e^{j \omega_{q}}\right)\right]=\phi_{q}, \quad q=1,2, \ldots, m .
$$

Since the constant term of $H_{m}(z)$ is zero, (24) indicates the presence of a linear phase component in the subset of phase values $\phi_{q}, q=1,2, \ldots, m$. One of the conditions for the algorithm to work is that the prescribed phase values should not contain a linear phase component. Since the recursion constructs a sequence of polynomials which satisfy a growing subset of the prescribed frequency/phase pairs, each subset also needs to satisfy the same condition. In the present situation where a given subset has a linear phase component but the complete set does not, the procedure will not work unless some remedial action is taken. Merchant (1988) suggested that the frequency/ phase pairs should be reordered by circulating them and then the recursion can be continued. 
Case 2:

But if

$$
\text { Phase }\left[P_{m}\left(e^{j \omega_{m}}\right)\right]=\phi_{m} .
$$

$$
\text { Phase }\left[P_{m-1}\left(e^{j \omega_{m}}\right)\right]=\phi_{m},
$$

then it implies that

$$
P_{m}(z)=P_{m-1}(z)
$$

Now, when $P_{m+1}(z)$ is computed, $C_{m+1}$ becomes 0 and recursion terminates. Again the frequencies can be circularly shifted as in case 1 and recursion continued.

However, it may happen that even after taking care of these situations, the recursion may terminate at a polynomial of order less than the desired order $N$ (Merchant 1988). This indicates the presence of a linear phase component in the full set of frequency-phase pairs which needs to be removed before the polynomial can be constructed. With premature termination, our program reports the last polynomial successfully constructed. As an example, consider the polynomial.

$$
P_{4}(z)=\left(1+z^{-1}+z^{-2}+z^{-3}\right)\left(1+4 z^{-1}\right) .
$$

Due to the presence of a symmetric factor in $P_{4}(z)$, recursion cannot proceed beyond the computation of $\boldsymbol{P}_{2}(z)$ (an intermediate polynomial).

However, it was observed that for

and for

$$
P_{2}(z)=\left(1+z^{-1}\right)\left(1+2 z^{-1}\right)
$$

$$
P_{3}(z)=\left(1-z^{-1}\right)\left(1+2 z^{-1}+3 z^{-2}\right)
$$

the presence of linear phase factors do not terminate the recursion and $P_{2}(z)$ and $P_{3}(z)$ are successfully constructed. It is inferred that zeros at $z= \pm 1$, though contributing to linear phase components, do not hinder the unique construction of a sequence from its phase values.

The design of our program is summarized in appendix A. To further validate the program, we consider a polynomial of order 6:

$$
P_{6}(z)=1+2 z^{-1}+3 z^{-2}+z^{-3}+4 z^{-4}+5 z^{-5}+z^{-6} \text {. }
$$

The phase values at an arbitrary set of frequencies are listed in table 1 . The coefficients obtained by implementing the procedure given here on the HP 9000 system are also shown in table 1 . The error in computed values, though negligible, is attributed to the finite-bit arithmetic of the computer.

\subsection{Explicit formulas for reconstruction}

In another recent contribution (Minocha et al 1990), we have derived explicit mathematical formulas for determining the coefficients of a polynomial (assuming the discrete signal to be real and of finite length) exactly, from the prescribed phase characteristics on the unit circle and have proposed an algorithm for the computation.

The explicit formulas have been obtained by solving the system of linear equations (6) for several low orders and then resorting to induction. It has been shown that

$$
p_{k}^{(N)}=-D_{k}^{(N)} / D^{(N)}, k=1,2, \ldots, N,
$$


Table 1. Comparison of the computed values of the coefficients with their actual values for the examples $[(27)$ and (34)].

\begin{tabular}{|c|c|c|c|c|c|}
\hline \multirow[b]{2}{*}{ Example } & \multirow[b]{2}{*}{$k$} & \multicolumn{2}{|r|}{ Specified } & \multicolumn{2}{|r|}{$p^{(N)}(k)$} \\
\hline & & $\begin{array}{l}\text { Frequency } \\
\text { (normalized) }\end{array}$ & $\begin{array}{c}\text { Phase } \\
\text { (radians) }\end{array}$ & Actual & Reconstructed \\
\hline \multirow[t]{6}{*}{ Equation (27) } & 1 & $\pi / 5$ & 0.859681459555361 & 2 & 1.999999996147184 \\
\hline & 2 & $\pi / 9$ & $-1 \cdot 208646365893548$ & 3 & 3.000000002299421 \\
\hline & 3 & $\pi / 10$ & -1.084476489414754 & 1 & 0.999999993685273 \\
\hline & 4 & $2 \pi / 5$ & $-4.5536120521889 E-002$ & 4 & 4000000002017118 \\
\hline & 5 & $2 \pi / 9$ & 0.546762582963989 & 5 & 4.999999993223931 \\
\hline & 6 & $3 \pi / 7$ & -0.470038925396254 & 1 & $1 \cdot 000000000361047$ \\
\hline \multirow[t]{4}{*}{ Equation (34) } & 1 & $\pi / 6$ & 0.534378199356056 & 1 & 1.00000000000002 \\
\hline & 2 & $\pi / 3$ & -1.047197653189843 & -2 & $-2 \cdot 00000000000000$ \\
\hline & 3 & $\pi / 2$ & $-1 \cdot 107148962577857$ & 3 & 3.0000000000004 \\
\hline & 4 & $2 \pi / 3$ & 0.132454424294178 & -4 & $-4 \cdot 00000000000006$ \\
\hline
\end{tabular}

Note: Due to monic polynomial condition, $p^{(N)}(0)=1$.

where $D_{k}^{(N)}$ and $D^{(N)}$ are given separately for two cases viz. $N=1,2$ and 3 (case 1) and $N \geqslant 4$ (case 2 ).

Case 1: $N=1,2 \& 3$

where

$$
D^{(N)}=\sum_{i=1}^{N} \sin \left(i \omega_{1}+\phi_{1}\right)\left\{\sum_{j=1}^{N-1+\delta_{N, 1}}(-1)^{r}\left[\prod_{q=1}^{N-1} \sin \left(L \omega_{q+1}+\phi_{q+1}\right)\right]\right\}
$$

$$
\begin{aligned}
\delta_{m, n} & \triangleq\left\{\begin{array}{l}
1, m=n, \\
0, m \neq
\end{array}\right. \\
r & =j+1+\delta_{i, 2} \delta_{N, 2}, \\
\prod_{m}^{n}(\cdot) & =1, n<m \\
L & =\left(\left(A+\delta_{A, i}\right)\right)_{N}^{\}}, \\
A & =((i+j-1+q))_{N}, \\
((m))_{N} & \triangleq\left\{\begin{array}{l}
m \text { modulo } N, m \neq n N, \\
N, m=n N, n \text { is an integer. }
\end{array}\right.
\end{aligned}
$$

The numerator, $D_{k}^{(N)}$, in (28) is also of the same form as $D^{(N)}$ with $i$ replaced by $i^{\prime}$ and $L$ replaced by $L^{\prime}$, where

and

$$
i^{\prime}=i\left(1-\delta_{i, k}\right)
$$

$$
L^{\prime}=L\left(1-\delta_{l, k}\right)
$$

Case 2: $N \geqslant 4$

$$
D^{(N)}=\sum_{i_{1}=1}^{N} \sin \left(a_{1} \omega_{1}+\phi_{1}\right) \cdot B \cdot C,
$$


where

and

$$
\begin{aligned}
B= & \left\{\sum _ { i _ { 2 } = i _ { 1 } + 1 } ^ { N + i _ { 1 } - 1 } \operatorname { s i n } ( a _ { 2 } \omega _ { 2 } + \phi _ { 2 } ) \left\{\sum_{i_{3}=i_{2}+1}^{N+i_{2}-2} \sin \left(a_{3} \omega_{3}+\phi_{3}\right) \cdots \times\right.\right. \\
& \left.\left.\times \sum_{i_{N-3}=i_{N-4}+1}^{N+i_{N-4}-N+4} \sin \left(a_{N-3} \omega_{N-3}+\phi_{N-3}\right)\right\} \cdots\right\} \\
C= & \sum_{j=1}^{3}\left[\prod_{q=N-2}^{N} \sin \left(a_{j m} \omega_{q}+\phi_{q}\right)-\prod_{q=N-2}^{N} \sin \left(b_{j m} \omega_{q}+\phi_{q}\right)\right],
\end{aligned}
$$

$$
m=(3-N+q) \text {. }
$$

The notations used in (32) will be clear from the algorithm discussed in appendix $B$.

As in case $1, D_{k}^{(N)}$ is of the same form as $D^{(N)}$ except for replacement of $a_{i}, i=1,2, \ldots, N$, by

$$
a_{i}^{\prime}=a_{i}\left(1-\delta_{a_{i}, k}\right)
$$

The formulas derived here [(29)-(33)], though seemingly involved, are easily implementable on a computer ${ }^{3}$. An efficient algorithm for computing $D^{(N)}, D_{k}^{(N)}$ and hence $p_{k}^{(N)}$ is described briefly in appendix $B$.

An example is now given to demonstrate the validity of the formulas and the algorithm.

Consider

$$
P_{4}(z)=1+z^{-1}-2 z^{-2}+3 z^{-3}-4 z^{-4} .
$$

The frequency/phase pairs along with the coefficients obtained by implementing the algorithm on an HP 9000 system are shown in table 1 .

Validation experiments show that the method should not be used for $N>10$ as it requires a rather large execution time.

\section{Conclusions}

In this section, we discuss some of the important situations where the phase interpolation problem arises and how the solution of the problem can be used to one's advantage.

In an FT. coding system, it is usual to transmit both magnitude and phase, after appropriate coding. If the signal satisfies the magnitude retrieval conditions, then it should suffice to transmit the coded phase only. A significant bit-rate reduction can be achieved thereby (Hayes et al 1980a).

In paleomagnetism (Oppenheim et al 1983), a history of the direction of the earth's magnetic field over time can be obtained by an examination of the core samples. This is, essentially, a phase information, and it would be very convenient if one can obtain the magnitude or strength information from this phase data.

Given the convolved output of a desired signal with some unknown distorting signal, the problem of blind deconvolution (Merchant 1982; Oppenheim et al 1983) involves the recovery of the desired signal, with no substantial information available about either the desired signal or the distorting signal. However, in the special case of a zero-phase distorting signal, the phases of the observed signal and the desired 
signal are identical; hence the desired signal can be reconstructed from phase information alone.

In a similar problem, signal reconstruction from only the FT phase can be useful in the estimation of the frequency response of a linear time-invariant system with some restrictions on the symmetry of the input to the system.

The role of phase-only reconstruction has also been exploited in the areas of image and speech processing (Fienup 1979; Oppenheim et al 1979; Oppenheim \& Lim 1981; Yegnanarayana et al 1984) and X-ray crystallography (Oppenheim \& Lim 1981). Images degraded by approximately zero-phase blurring functions (e.g. by defocused lenses in an optical system) can be reconstructed from the knowledge of phase alone as the phase of the blurred image would be similar to that of the original image. With FT magnitude assumed to be unity, it has been shown that the intelligibility of speech is retained in a phase-only reconstruction. In the area of X-ray crystallography, it has been observed that the atomic structure, inferred from the diffraction data, is better preserved in a magnitude-retrieval Fourier synthesis than in a phase-retrieval Fourier synthesis.

\section{Appendix A}

A flow chart of the main program module is given in figure A1.

Following this module, we use a less formal method of stating the algorithm.

(1) The recursion can be initiated only for $N \geqslant 1$. The program checks the value of $\mathbf{N}$ and reads the given frequency/phase pairs.

(2) Initialize: $m=1 \& k=0 ; m$ is the order of the polynomial being computed in the sequence of polynomials $P_{m}(z), m=1,2, \ldots, N$ and $k$ is the parameter that controls the circular shift of the frequency/phase pairs

(3) 3.1 If

3.2 Then

$\left(\omega_{1}+\phi_{1}\right) / \pi=0$ or any integer

3.2a CALL subroutine SHIFT

$3.2 b$

SHIFT:

If $\quad K=N-m+1$

Then it indicates that while circularly shifting the frequencies all the frequency/phase pairs $\left(\omega_{q}, \phi_{q}\right), q=m+1, \cdots, N$ have been tried; recursion prematurely terminates with a message

Else increment $k$, circularly shift the frequencies. Control passes to main program.

3.2c Go to step 3.1.

3.3 Else Compute $p^{(1)}(1)$.

(4) CALL subroutine OUTPUT

OUTPUT prints $p^{(m)}(r), \quad r=1,2, \ldots, m$

(5) Initialize: $k=0$; Increment $m$.

If $\quad m=N+1$

Then it means that the desired $N$ th order polynomial has been determined and therefore recursion stops

Else (A) CALL subroutine CHECK

CHECK takes care of cases $1 \& 2$ and their remedies discussed in $\S 4.1$. 


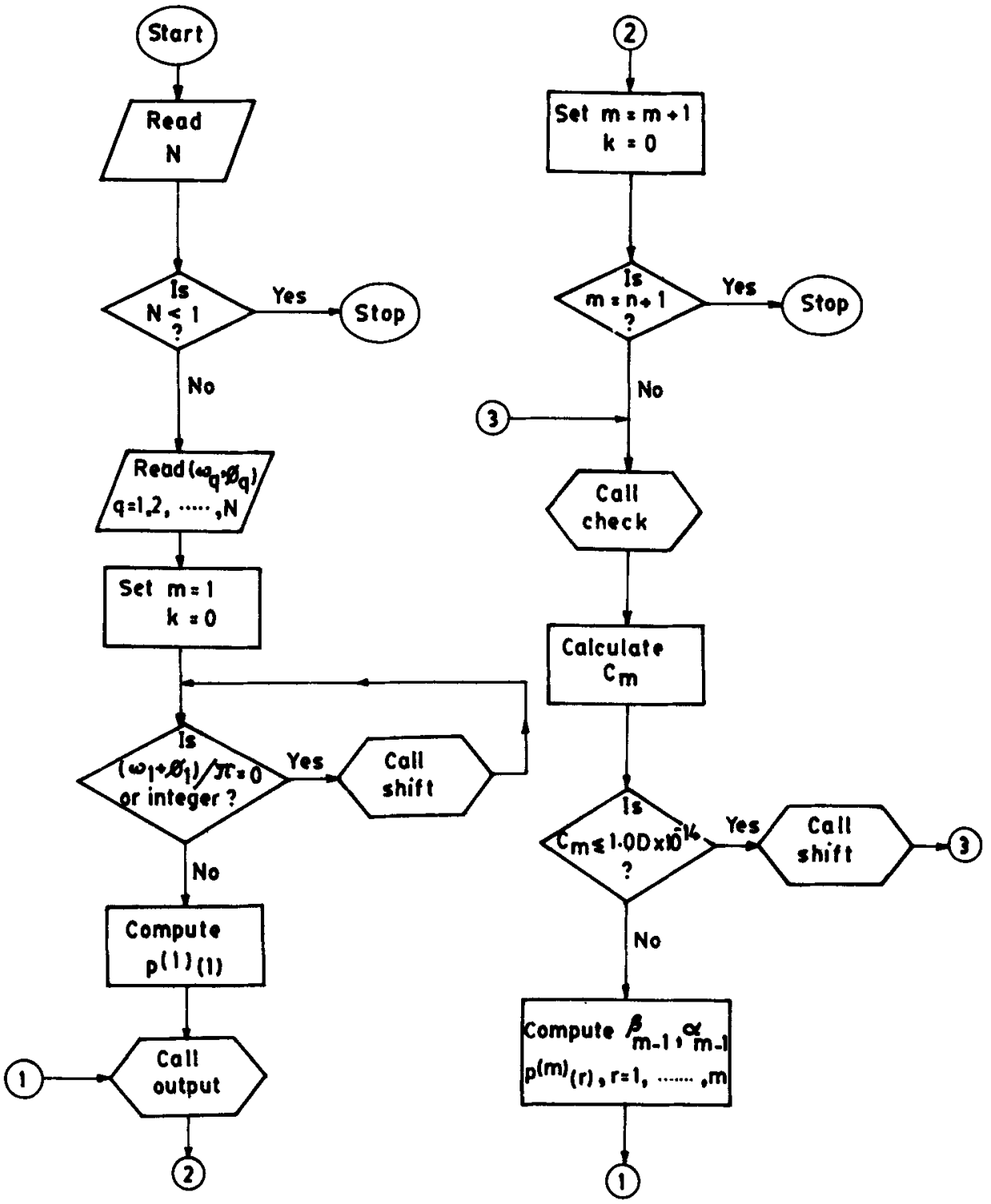

Figure A1. Flow chart of the main program module for the implementation of Merchant's (1988) recursion.

(B) Compute $C_{m}$

(C) If $C_{m} \leqslant 1.0 \mathrm{D} 0 \times 10^{-14} \Rightarrow C_{m} \approx 0$

Then (i) CALL subroutine SHIFT. SHIFT checks $k$ and reorders the frequency/phase pairs as in step $3.2 \mathrm{~b}$

(ii) Go to (A).

\section{Else Compute}

(6)

$\beta_{m-1}, \alpha_{m-1}$ and $p^{(m)}(r), \quad r=1,2, \ldots, m$

Go to 4 . 


\section{Appendix B}

The explicit formulas derived for orders $\mathbf{N}=1,2$ \& 3 [(29)-(31)] can be easily programmed and implemented. For orders $N \geqslant 4$, the computer program implementing (32) and (33) to determine $p_{k}^{(N)}, k=1,2, \ldots, N$ is organized as follows:

Main program computes $p_{k}^{(N)}$ using (28).

The subprograms are as follows:

(i) For evaluating denominator, $D^{(N)}$.

(ii) For evaluating the numerator, $D_{k}^{(N)}$.

(iii) For negating the sign of the product term, each time the index of any summation increments by 1 .

(iv) For computing the Kronecker delta [(30a)].

(v) For computing the modulo [(30f)].

(vi) For sorting the array in ascending order.

(viii) The major task in evaluating $D^{(N)}$ or $D_{k}^{(N)}$ is to determine the coefficients, $a_{n}, n=1,2, \ldots, N$ for the products of the sine terms to be generated. $a_{n}$ 's are computed in a subprogram using the subprograms (iv)-(vi) by the following method:

$$
a_{n}=\left\{\begin{array}{l}
\left(\left(i_{n}\right)_{N}, \quad n=1,2,\right. \\
\left(\left(d_{n, n-1}\right)\right)_{N}^{!}, \quad n=3,4, \ldots, N,
\end{array}\right.
$$

where $d_{n, n-1}$ is computed as follows. Let $c_{i}, i=1,2, \ldots, n-1$ be the array in which the $a_{i}$ 's are arranged in ascending order when $a_{n}$ is being evaluated.

Then let

$$
p=\left(\left(a_{n-1}+1\right)\right)_{N}, \text { when } i_{j}=i_{j-1}+1,
$$

i.e. at the start of the loop; else

and

$$
\begin{aligned}
& p=\left(\left(a_{n}+1\right)\right)_{N}, \\
& d_{n, 1}=((p))_{N}^{l}+\delta_{((p))_{N}, c_{1}},
\end{aligned}
$$

$$
d_{n, m}=\left(\left(d_{n, m-1}\right)\right)_{N}+\delta_{\left(\left\{d_{n, m-1}\right)\right\}_{,}, c_{m}}, m=2,3, \ldots, n-1,
$$

$d_{n, n-1}$ is substituted in (B1) to get $a_{n}$. The $a_{n}, n=1,2, \ldots, N-3$ are used in (32a) and (32b) and for the innermost summation, (32c), the following substitutions are made:

$$
\begin{aligned}
& a_{1,3-N+q}=a_{q}, \quad q=N-2, N-1, N, \\
& a_{j, 3-N+q}=a_{1, j 1}, \quad j=2,3,
\end{aligned}
$$

where $j 1$ is defined as

Also

$$
j 1=(4-N+q)-(3-N+q) \delta_{3-N+q, 3} .
$$

$$
\begin{aligned}
b_{j, 1} & =a_{j, 1}, \\
b_{j, 2} & =a_{j, 3}, \\
b_{j, 3} & =a_{j, 2}, \quad j=1,2,3 .
\end{aligned}
$$

By repeated usage of subprogram (vii), $D^{(N)}$ and $D_{k}^{(N)}$ are evaluated, yielding $p_{k}^{(N)}$. 


\section{References}

Akaike H 1973 Block Toeplitz matrix inversion. Siam J. Appl. Math. 24: 234-241

Fienup J R 1978 Reconstruction of an object from the modulus of its Fourier transform. Opt. Lett. 3: 27-29

Fienup J R 1979 Space object imaging through the turbulent atmosphere. Opt. Eng. 18: 529-534

Gerchberg R W, Saxton W O 1972 A practical algorithm for the determination of phase from image and diffraction plane pictures. Optik 35: 237-246

Hayes M H 1982 The reconstruction of a multidimensional sequence from the phase or magnitude of its Fourier transform. IEEE Trans. Acoust., Speech Signal Process. ASSP-30: 140-154

Hayes M H, Lim J S, Oppenheim A V 1980a Signal reconstruction from phase or magnitude. IEEE Trans. Acoust., Speech Signal Process. ASSP-28: 672-680

Hayes M H, Lim J S, Oppenheim A V 1980b Phase-only signal reconstruction, Proc. ICASSP (New York: IEEE Press) pp. 437-440

Madhu Murthy K V, Yegnanarayana B 1989 Effectiveness of representation of signals through group delay functions. Signal Process. 17: 141-150

Merchant G A 1982 Reconstruction of signals from phase: Efficient algorithms, segmentation, and generalization, Ph D Thesis, Rice University, Houston, Texas

Merchant G A 1988 Recursive construction of polynomial with prescribed phase on unit circle. IEEE Trans. Acoust., Speech Signal Process. ASSP-36: 1531-1533

Merchant G A, Parks T W 1982 Efficient solution of a Toeplitz-plus-Hankel coefficient matrix system of equations. IEEE Trans. Acoust., Speech Signal Process. ASSP-30: 40-44

Merchant G A, Parks T W 1983 Reconstruction of signals from phase: Efficient algorithm, segmentations, and generalizations. IEEE Trans. Acoust., Speech Signal Process. ASSP-31: 1135-1147

Minocha S, Dutta Roy S C, Kumar B 1990 Explicit formulas for reconstruction of a discrete signal from the phase of its Fourier transform. IEEE Trans. Circuits Syst. (submitted)

Minocha S, Dutta Roy S C, Kumar B 1991 Recursive construction of polynomial with prescribed phase on unit circle Electron. Lett. 27: 224-225

Oppenheim A V 1981 Signal reconstruction for Fourier transform or magnitude. Workshop on Digital Signal Processing, Italy, CNR, Italy and NSF, USA, pp. 131-145

Oppenheim A V, Hayes M H, Lim J S 1982 Iterative procedures for signal reconstruction from Fourier transform phase. Opt. Eng. 21: 122-127

Oppenheim A V, Lim J S 1981 The importance of phase in signals. Proc. IEEE 69: 529-541

Oppenheim A V, Lim J S, Curtis S R 1983 Signal synthesis and reconstruction from partial Fourier-domain information. J. Opt. Soc. Am. 73: 1413-1420

Oppenheim A V, Lim J S, Kopec G, Pohling S C 1979 Phase in speech and pictures. Proc. ICASSP (New York: IEEE Press) pp. 634-637

Oppenheim A V, Schafer R W 1975 Digital signal processing (Englewood Cliffs, NJ: Prentice-Hall)

Quatieri T R, Oppenheim A V 1981 Iterative techniques for minimum phase signal reconstruction from phase or magnitude. IEEE Trans. Acoust., Speech Signal Process. ASSP- 29: 1187-1193

Tom V T, Quatieri T R, Hayes M H, McClellan J H 1981 Convergence of iterative nonexpansive signal reconstruction algorithms. IEEE Trans. Acoust., Speech Signal Process. ASSP- 29: 1052-1058

Tribolet J M 1977 A new phase unwrapping algorithm. IEEE Trans. Acoust., Speech Signal Process. ASSP25: $170-177$

Van Hove P L, Hayes M H, Lim J S, Oppenheim A V 1983 Signal reconstruction from signed Fourier transform magnitude. IEEE Trans. Acoust., Speech Signal Process. ASSP-31: 1286-1293

Yegnanarayana B 1981a Speech analysis by pole-zero decomposition of short-time spectra. Signal Process. 3: 5-17

Yegnanarayana B $1981 \mathrm{~b}$ Design of ARMS digital filtering by pole-zero decomposition. IEEE Trans. Acoust., Speech Signal Process. ASSP-29: 433-439

Yegnanarayana B 1982 Design of recursive group-delay filters by autoregressive modelling. IEEE Trans. Acoust., Speech Signal Process. ASSP-30: 632-637

Yegnanarayana B, Dhayalan A 1983 Noniterative techniques for minimum phase signal reconstruction from phase or magnitude. Proc. ICASSP (New York: IEEE Press) pp. 639-642

Yegnanarayana B, Duncan G, Murthy H A 1988b Formant extraction from group delay function. Proc IEE Colloquium on Signal Processing, London

Yegnanarayana B, Madhu Murthy K V, Murthy H A 1988a Applications of group delay functions in speech processing. J. Inst. Elect. Telecommun. Eng. 34: 20-29 
Yegnanarayana B, Mariadassou C P, Saini Pramod 1990 Signal reconstruction from partial data for sensor array imaging applications. Signal Process. 19: 139-149

Yegnanarayana B, Murthy H A 1990 Spectrum estimation using Fourier transform phase. Proc. Workshop Signal Processing Communications \& Networking, Bangalore pp. 44-53

Yegnanarayana B, Saikia D K, Krishnan T R 1984 Significance of group delay functions in signal reconstruction from spectral magnitude or phase. IEEE Trans. Acoust., Speech Signal Process. ASSP- 32: 610-622 